\title{
ON A CONJECTURE CONCERNING HELLY CIRCLE GRAPHS
}

\author{
Guillermo Durán, Agustín Gravano, Marina Groshaus \\ Universidad de Buenos Aires - Pabellón I \\ Buenos Aires - Argentina \\ willy@dc.uba.ar, agravano@dc.uba.ar, groshaus@dc.uba.ar
}

Fábio Protti*, Jayme L. Szwarcfiter

Universidade Federal do Rio de Janeiro

Rio de Janeiro - RJ - Brasil

fabiop@nce.ufrj.br, jayme@nce.ufrj.br

* Corresponding author/autor para quem as correspondências devem ser encaminhadas

Recebido em 06/2002, aceito em 11/2002 após 1 revisão

\begin{abstract}
We say that $G$ is an e-circle graph if there is a bijection between its vertices and straight lines on the cartesian plane such that two vertices are adjacent in $G$ if and only if the corresponding lines intersect inside the circle of radius one. This definition suggests a method for deciding whether a given graph $G$ is an e-circle graph, by constructing a convenient system $S$ of equations and inequations which represents the structure of $G$, in such a way that $G$ is an e-circle graph if and only if $S$ has a solution. In fact, e-circle graphs are exactly the circle graphs (intersection graphs of chords in a circle), and thus this method provides an analytic way for recognizing circle graphs. A graph $G$ is a Helly circle graph if $G$ is a circle graph and there exists a model of $G$ by chords such that every three pairwise intersecting chords intersect at the same point. A conjecture by Durán (2000) states that $G$ is a Helly circle graph if and only if $G$ is a circle graph and contains no induced diamonds (a diamond is a graph formed by four vertices and five edges). Many unsuccessful efforts - mainly based on combinatorial and geometrical approaches - have been done in order to validate this conjecture. In this work, we utilize the ideas behind the definition of e-circle graphs and restate this conjecture in terms of an equivalence between two systems of equations and inequations, providing a new, analytic tool to deal with it.
\end{abstract}

Keywords: circle graph; Helly circle graph.

\section{Resumo}

Dizemos que $G$ é um grafo e-circular se existe uma bijeção entre seus vértices e retas no plano cartesiano de forma que dois vértices são adjacentes em $G$ se e somente se as retas correspondentes se intersectam dentro do círculo de raio unitário centrado na origem. Esta definição sugere um método para decidir se um dado grafo $G$ é um grafo e-circular, construindo convenientemente um sistema $S$ de equações e inequações que representa a estrutura de $G$, de tal modo que $G$ é um grafo e-circular se e somente se $S$ tem solução. Em realidade, grafos e-circulares são exatamente os grafos circulares (grafos de interseção de cordas em um círculo), e portanto este método fornece um modo analítico de reconhecimento de grafos circulares. Um grafo $G$ é circular Helly se $G$ é um grafo circular e existe um modelo de cordas de $G$ tal que em todo grupo de três cordas mutuamente intersectantes existe um ponto comum a todas elas. Uma conjectura de Durán (2000) afirma que $G$ é um grafo circular Helly se e somente se $G$ é um grafo circular e não contém diamantes induzidos (um diamante é um grafo formado por quatro vértices e cinco arestas). Muitas tentativas infrutíferas - baseadas principalmente em abordagens combinatórias e geométricas - foram realizadas para tentar validar a conjectura. Neste trabalho, utilizamos as idéias subjacentes à definição de grafos e-circulares e reformulamos a conjectura em termos de uma equivalência entre dois sistemas de equações e inequações, fornecendo uma nova ferramenta analítica para tratá-la.

Palavras-chave: grafo circular; grafo circular Helly. 


\section{Introduction}

Let $G=(V(G), E(G))$ be a graph, and $n=|V(G)|$. We say that $G$ is a circle graph if there exists a family of chords $L$ on a circle and a one-to-one correspondence between vertices of $G$ and chords of $L$ such that two distinct vertices are adjacent in $G$ if and only if the corresponding chords in $L$ intersect. Such a family of chords representing $G$ is called a chord model for $G$. Circle graphs first appeared in Even \& Itai (1971), in the context of a problem proposed in Knuth (1969). Polynomial time recognition algorithms for circle graphs can be found in Bouchet (1987), Gabor, Supowit \& Hsu (1989) and Spinrad (1994).

Helly circle graphs (Durán, 2000) form a natural subclass of circle graphs. We say that $G$ is a Helly circle graph if there exists a chord model $L$ for $G$ satisfying the Helly property. (A family $L$ of chords satisfies the Helly property when every subfamily of pairwise intersecting chords of $L$ has a common point of intersection.) A conjecture by Durán (2000) states that $G$ is a Helly circle graph if and only if $G$ is a circle graph and contains no induced diamonds (a graph formed by four vertices and five edges.)

Many unsuccessful efforts, mainly based on combinatorial and geometrical approaches, have been done in order to validate this conjecture. In this work, we define the class of $e$-circle graphs - which is shown to be in fact exactly the class of circle graphs - and we utilize the ideas behind this definition to restate this conjecture in terms of an equivalence between two systems of equations and inequations, providing a new, analytic tool to deal with it.

\section{E-circle graphs}

A graph $G$ is an e-circle graph if we can find a model of straight lines on the cartesian plane according to the following procedure.

Represent each vertex $v \in V(G)$ by a line $R_{v}$, fixing the origin of coordinates at the center of the circle of radius $r=1$. Two lines intersect inside the circle if and only if their respective vertices are adjacent in $G$ (Figure 1). Let $R(G)$ be the set of lines corresponding to the vertices of $G$.

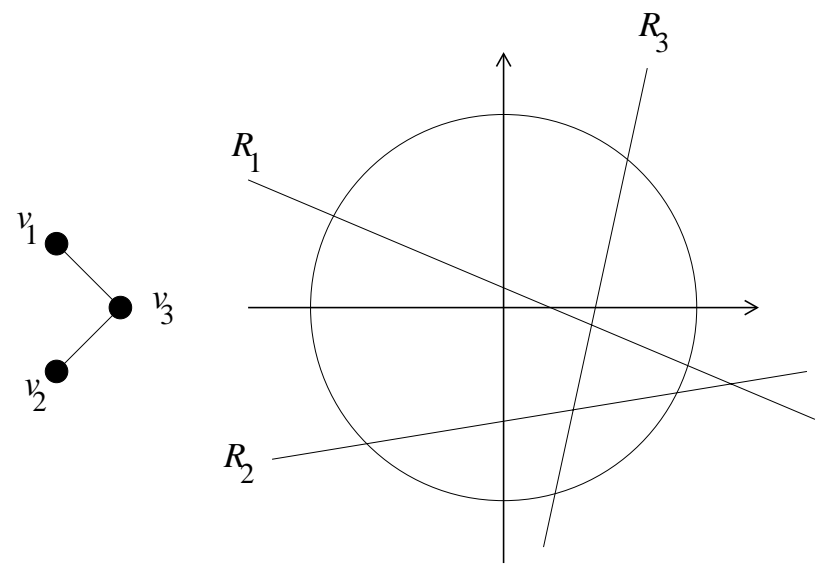

Figure 1 - System of lines for a three-vertex graph. 
To formalize this concept, we make the following simplifications:

- There are no vertical lines in $R(G)$.

- There are no horizontal lines in $R(G)$.

- There are no pairs of parallel lines in $R(G)$.

Let us verify that these simplifications do not imply a loss of generality. Suppose that a graph $G$ is represented by a set of lines $R(G)$ such that for every pair of vertices $i, j \in G$ with $i \neq j, R_{i}$ intersects $R_{j}$ inside the circle if and only if $i$ is adjacent to $j$ in $G$. If there are any vertical or horizontal lines in $R(G)$, they can be rotated slightly, until there are no vertical and horizontal lines left. If there is a pair of parallel lines, one of them can be rotated slightly in order to eliminate that parallelism, and without modifying the crossings that take place inside the circle.

The first simplification allows each straight line $R_{i}$ to be written as an equation $y=m_{i} \cdot x+b_{i}$. The second and third ones guarantee that $\forall i, m_{i} \neq 0$, and $\forall i \neq j, m_{i} \neq m_{j}$.

As said before, fix the origin of coordinates at the center of the circle of radius $r=1$, and construct a system of equations and inequations in the following way.

For each pair of vertices $i, j \in V(G)(i \neq j)$, add the following equations:

- $y_{\langle i, j\rangle}=m_{i} \cdot x_{\langle i, j\rangle}+b_{i}$

- $y_{\langle i, j\rangle}=m_{j} \cdot x_{\langle i, j\rangle}+b_{j}$

where $\langle i, j\rangle$ is the point where lines $R_{i}$ and $R_{j}$ intersect, and $x_{\langle i, j\rangle}, x_{\langle i, j\rangle}$, are its coordinates. Add also the following inequations:

- $x_{\langle i, j\rangle}^{2}+y_{\langle i, j\rangle}^{2}<1 \quad$ if the edge $(i, j) \in E(G)$

- $x_{\langle i, j\rangle}^{2}+y_{\langle i, j\rangle}^{2}>1 \quad$ otherwise

That is, lines $R_{i}$ and $R_{j}$ intersect inside the circle if and only if vertices $i$ and $j$ are adjacent in $G$. Since there cannot be any pair of parallel lines, they must intersect somewhere, either inside or outside the circle. The case in which $x_{\langle i, j\rangle}^{2}+y_{\langle i, j\rangle}^{2}=1$ can be ignored: if there is a model $M$ of lines in which this equality holds, $M$ can be slightly modified in order to transform the equality into a strict inequality.

Finally, add the following restrictions to the system, as a consequence of simplifications 2 and 3:

- $m_{i} \neq 0 \quad \forall i \in V(G)$

- $m_{i} \neq m_{j} \quad \forall i, j \in V(G)$, with $i \neq j$.

The number of equations and inequations is $2 n^{2}-2$. An e-circle graph is formally defined as a graph for which the system of equations and inequations constructed in this way has a solution. 
Theorem 1. A graph $G$ is an e-circle graph if and only if it is a circle graph.

Just observe that any pair of parallel chords can be moved slightly eliminating thus the parallelism without affecting other intersections. The proof of Theorem 1 follows directly.

\section{The Diamond's Conjecture}

A graph $G$ is a diamond if it is isomorphic to $K_{4}-\{e\}$. Durán (2000) observed the following property:

Remark 1. Let $G$ be a Helly circle graph. Then $G$ is a circle graph and contains no induced diamonds.

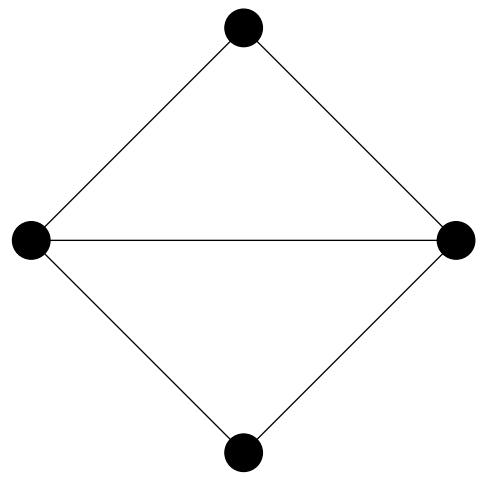

Figure 2 - A diamond

In the same work, Durán conjectured that the converse of this property is also true:

Conjecture 2. Let $G$ be a circle graph containing no induced diamonds. Then $G$ is a Helly circle graph.

This interesting conjecture remains open. Note that if it were true, it would give us a polynomial recognition algorithm for Helly circle graphs. Here, we show a reformulation of this conjecture, using the ideas just presented.

Let $G=(V(G), E(G))$ be any graph. First, construct a system $S_{G}$ of equations and inequations for $G$, following the rules explained above. As we have already seen, $S_{G}$ has a solution if and only if $G$ is a circle graph. In order to complete the reformulation of Conjecture 2, we need to be able to express two more facts analytically: that $G$ contains no induced diamonds, and that $G$ is a Helly circle graph. We will use the following notations:

- Given a model of straight lines on the plane for a graph $G$, and two vertices $i, j \in V(G)$, let $\langle i, j\rangle$ denote the point in which the corresponding lines intersect.

- $x_{\langle i, j\rangle}$ and $y_{\langle i, j\rangle}$ are the coordinates of the point $\langle i, j\rangle$. 
- $\|\langle i, j\rangle\|$ is the distance from the point $\langle i, j\rangle$ to the origin of coordinates. That is, $\|\langle i, j\rangle\|=\sqrt{x_{\langle i, j\rangle}^{2}+y_{\langle i, j\rangle}^{2}}$.

- Given $a, b \in \mathfrak{R}, a-b=\left\{\begin{array}{lll}a-b & \text { if } & \mathrm{a} \geq \mathrm{b} \\ 0 & \text { if } & \mathrm{a}<\mathrm{b} .\end{array}\right.$

Note that $a-b \geq 0, \forall a, b \in \mathfrak{R}$.

\subsection{Expressing that $G$ contains no induced diamonds}

In this subsection we show how to express analytically the fact that $G$ contains no induced diamonds.

For each set $U$ of four vertices in $V(G)$, add the following inequations to system $S_{G}$ :

- For each $i, j \notin U$, such that $i \neq j$ :

$$
\delta_{U}^{0} \leq 1 \bullet\|\langle i, j\rangle\|
$$

- For every possible combination of $\{\{i, j\},\{k, l\}\} \subseteq \mathrm{P}(U)$, such that $i \neq j, k \neq l$, and $\{i, j\} \neq\{k, l\}$

$$
\begin{aligned}
& \delta_{U}^{i j, k l} \leq\|\langle i, j\rangle\|-1 \\
& \delta_{U}^{i j, k l} \leq\|\langle k, l\rangle\|-1
\end{aligned}
$$

- Finally:

$$
\delta_{U}^{0}+\sum_{\substack{\{i, j\},\{k, l\}\}, i \neq j, k \neq l \\\{i, j\} \neq\{k, l\}}} \delta_{U}^{i j, k l}>0
$$

where $\delta_{U}^{0}, \delta_{U}^{i j, k l} \in \mathfrak{R}$. For a graph $G$, with $n \geq 4$, there are $\left(\begin{array}{l}n \\ 4\end{array}\right)$ distinct subsets of four vertices in $V(G)$. Therefore, the total number of inequations that are added to the system is $\mathrm{O}\left(n^{4}\right)$.

Proposition 3. The new system $S_{G}^{\prime}$ constructed from a graph $G$ has a solution if and only if $G$ is a circle graph and contains no induced diamonds.

Proof: First, suppose that the system $S_{G}^{\prime}$ has a solution. Recall from last section that if the original system $S_{G}$ has a solution then $G$ is a circle graph. We have to prove that if the new inequations can be satisfied then $G$ contains no induced diamonds. 
For each set $U$ of four vertices in $V(G)$, at least one of the two terms of Inequation (3) must be positive:

- If $\delta_{U}^{0}>0$, then, due to Inequations (1), the lines corresponding to the vertices in $U$ intersect each other inside the circle. This means that the subgraph induced by $U$ is complete, and not a diamond.

- If the sum of Inequations (3) is positive, then it is true that $\delta_{U}^{i j, k l}>0$ for some combination of $\{\{i, j\},\{k, l\},\{k, l\}\} \subseteq \mathrm{P}(U)$, such that $i \neq j, k \neq l$. Inequations (2) imply that the points $\langle i, j\rangle$ and $\langle k, l\rangle$ lay outside the circle. So, $G$ does not contain the two corresponding edges, and this guarantees that the subgraph induced by $U$ is not a diamond.

We have just seen that if the system has a solution, then $G$ is a circle graph and any subgraph induced by four vertices in $G$ is either complete or contains at most four edges. In other words, if $S_{G}$ has a solution, then $G$ is a circle graph containing no induced diamonds.

Suppose now that $G$ is a circle graph and contains no induced diamonds. We know that the original system $S_{G}$ has a solution because $G$ is a circle graph. Let us see that the new inequations are satisfied for some choice of $\delta^{\prime}$ s.

Let $U$ be a set of four vertices in $V(G)$ :

- If the subgraph induced by $U$ is complete, choose:

$$
\begin{aligned}
& \delta_{U}^{0}=\min _{i j \in U}(\dot{-}\|\langle i, j\rangle\|) \\
& \delta_{U}^{i j, k l}=0 \quad \forall\{\{i, j\},\{k, l\}\} \subseteq \mathrm{P}(U): i \neq j, k \neq l,\{i, j\} \neq\{k, l\}
\end{aligned}
$$

As the subgraph induced by $U$ is complete, the lines representing its vertices cross each other strictly inside the circle, according to the solution of system $S_{G}$. Therefore, the value chosen for $\delta_{U}^{0}$ is greater than zero, and satisfies the inequations of $S_{G}^{\prime}$.

- If the subgraph induced by $U$ is not complete, then it contains at most four edges, since $G$ contains no induced diamonds. In this case, choose:

$$
\begin{aligned}
\delta_{U}^{0} & =c \\
\delta_{U}^{i j, k l} & = \begin{cases}0 & \text { if }\{(i, j),(k, l)\} \cap E(G) \neq 0 \\
\min \{\|\langle i, j\rangle\|,\|\langle k, l\rangle\|\} \dot{-} & \text { if }\{(i, j),(k, l)\} \cap E(G)=0\end{cases}
\end{aligned}
$$

The last value corresponds to the case in which the edges $(i, j)$ and $(k, l)$ are not in the graph. Then, the intersections of the corresponding lines lay outside the circle, which guarantees that $\delta_{U}^{i j, k l}>0$. 


\subsection{Expressing that $G$ is a Helly circle graph}

In this section, we show how to express analytically that $G$ is a Helly circle graph.

For each set $U$ formed by three vertices in $V(G)$, add the following inequations to the original system $S \_G$ :

$$
\begin{gathered}
\sum_{\substack{i, j \in U \\
i \neq j}}(\|\langle i, j\rangle\| \dot{-1}) \leq \eta_{U} \cdot c \\
\sum_{\substack{i, j \in U \\
i \neq j}}\left(x_{\langle i, j\rangle} \dot{-} \alpha_{U}\right) \leq \eta_{U} \cdot c \\
\sum_{\substack{i, j \in U \\
i \neq j}}\left(y_{\langle i, j\rangle} \dot{-} \beta_{U}\right) \leq \eta_{U} \cdot c \\
\sum_{\substack{i, j \in U \\
i \neq j}}\left(\alpha_{U} \dot{-} x_{\langle i, j\rangle}\right) \leq \eta_{U} \cdot c \\
\sum_{\substack{i, j \in U \\
i \neq j}}\left(\beta_{U} \dot{-} y_{\langle i, j\rangle}\right) \leq \eta_{U} \cdot c \\
-\sum_{\substack{i, j \in U \\
i \neq j}}(\|\langle i, j\rangle\| \dot{-1}) \leq\left(1-\eta_{U}\right) \cdot c \\
c=\max _{\substack{i, j \in V(G) \\
i \neq j}}\|\langle i, j\rangle\| \cdot 3+1
\end{gathered}
$$

Where $\eta_{U}, \alpha_{U}, \beta_{U}, c \in \mathfrak{R} ; \quad \eta_{U} \in\{0,1\}$. For a graph $G$ with $n \geq 3$, there are $\left(\begin{array}{l}n \\ 3\end{array}\right)$ distinct subsets of three vertices in $V(G)$. Therefore, the total number of inequations that are added to the system is $\mathrm{O}\left(n^{3}\right)$.

Proposition 4. The new system $S_{G}^{\prime \prime}$ constructed from a graph $G$ has a solution if and only if $G$ is a Helly circle graph.

Proof: Let $G=(V(G), E(G))$ be any graph. First, suppose that $S_{G}^{\prime \prime}$ has a solution. As we have already seen, if the original system $S_{G}$ has a solution then $G$ is a circle graph. We have to prove that if the new inequations are satisfied, then the model of lines for $G$ verifies the Helly property. 
Let $U$ be a set formed by three vertices in $V(G)$. We know that Inequations (4) to (10) are satisfied. $\eta_{U}$ can take two different values: 0 or 1 . Suppose that $\eta_{U}=0$. Then:

- Inequations (4) imply that $\|\langle i, j\rangle\| \leq 1$ for all $\langle i, j\rangle$, which means that $U$ is complete.

- Inequations (5) and (7) imply that $\alpha_{U}=x_{\langle i, j\rangle}$ for all $\langle i, j\rangle$.

- Inequations (6) and (8) imply that $\beta_{U}=y_{\langle i, j\rangle}$ for all $\langle i, j\rangle$.

Therefore, for every complete subgraph $U$ with three vertices, its corresponding lines cross at the same point (at coordinates $x=\alpha_{U}$, and $x=\beta_{U}$ ).

Let us now analyze the case $\eta_{U}=1$. Inequations (9) say that there exists at least one $\langle i, j\rangle$ such that $\|\langle i, j\rangle\|>1$. Thus, $U$ is not complete.

We have just seen that for every set $U$ of three vertices, if the subgraph induced by $U$ is complete, then its lines cross each other at the same point. This means that all the lines in every complete subgraph of $G$ (of any size) cross at the same point. Therefore, the model of lines defined by system $S_{G}^{\prime \prime}$ verifies the Helly property.

Now suppose that $G$ is a Helly circle graph. We know that the original system $S_{G}$ has a solution because $G$ is a circle graph. Let us see that the new inequations are satisfied for some choice of values for the variables.

Again, let $U$ be a set of three vertices in $V(G)$. If the subgraph induced by $U$ is complete, choose:

- $\eta_{U}=0$

- $\alpha_{U}$ and $\beta_{U}$ equal to coordinates $x$ and $y$, respectively, of the point where all lines corresponding to vertices in $U$ cross, according to the solution of system $S_{G}$

Right terms of Inequations (4) to (8) are equal to 0 , thus making left terms also equal to 0 . In the first case (Inequations (4)), that equality is true because $\|\langle i, j\rangle\|>1$ for all $\langle i, j\rangle$ (provided that $U$ is complete). In the other cases, the equalities hold because coordinates $x$ and $y$ of all the $\langle i, j\rangle$ are $\alpha_{U}$ and $\beta_{U}$, respectively. Finally, in order to verify Inequations (9), note that the only possible value of $c$ is greater than 0 .

If the subgraph induced by $U$ is not complete, choose:

- $\eta_{U}=1$

- $\alpha_{U}=\beta_{U}=0$

In this case, Inequations (9) are satisfied because there must exist some $\langle i, j\rangle$ such that $\|\langle i, j\rangle\|>1$, since $U$ is not complete. Finally, it is easy to see that Inequations (4) to (8) are satisfied, because the only possible value of $c$ is an upper bound for each sum. 


\subsection{Restating the Diamond's Conjecture}

By using Propositions 3 and 4, Conjecture 2 can be restated as follows:

Conjecture 5. Let $G$ be any graph. If the system of inequations $S_{G}^{\prime}$ has a solution, then system $S_{G}^{\prime \prime}$ also has a solution.

According Remark 1, the converse is true.

\section{Acknowledgements}

Guillermo Durán was partially supported by UBACyT Grants X036 and X127, and PID Conicet Grant 644/98, Argentina.

Agustín Gravano was partially supported by UBACyT Grant X127, Argentina.

Marina Groshaus was partially supported by UBACyT Grant X036, Argentina.

Fábio Protti was partially supported by Conselho Nacional de Desenvolvimento Científico e Tecnológico - CNPq, Brasil.

Jayme L. Szwarcfiter was partially supported by the Conselho Nacional de Desenvolvimento Científico e Tecnológico - CNPq, and Fundação de Amparo à Pesquisa do Estado do Rio de Janeiro - FAPERJ, Brasil.

\section{References}

(1) Bouchet, A. (1987). Reducing prime graphs and recognizing circle graphs. Combinatorica, 7(3), 243-254.

(2) Durán, G. (2000). On Intersection Graphs of Arcs and Chords in a Circle. Doctoral Thesis, Universidad de Buenos Aires, Argentina, In Spanish.

(3) Even, S. \& Itai, A. (1971). Queues, stacks and graphs. In: Theory of Machines and Computations [edited by A. Kohavi and A. Paz], Academic Press, New York, 71-86.

(4) Gabor, C.; Supowit, K. \& Hsu, W. (1989). Recognizing circle graphs in polynomial time. Journal of the ACM, 36(3), 435-473.

(5) Knuth, D. (1969). The Art of Computer Programming. Vol. 1, Addison-Wesley.

(6) Spinrad, J. (1994). Recognition of circle graphs. Journal of Algorithms, 16(2), 264-282. 